\title{
Chapter 20 \\ Enhancement of Bone Tissue Repair by Octacalcium Phosphate Crystallizing into Hydroxyapatite In Situ
}

\author{
Osamu Suzuki and Takahisa Anada
}

\begin{abstract}
We have reported that octacalcium phosphate (OCP) enhances bone repair in critical-sized rat calvaria defects, while OCP is gradually crystallized to apatite structure. Our studies showed that:
\end{abstract}

1. OCP enhances differentiation of osteoblastic cells in two- and three-dimensional conditions.

2. OCP enhances osteoclast formation from bone marrow cells (macrophages) in the co-culture with osteoblasts by raising osteoclast-inducing factor (RANKL).

3. OCP enhances macrophages migration.

4. These cellular responses are brought about associated with the hydrolysis of OCP toward a nonstoichiometric Ca-deficient hydroxyapatite (HA), which is accompanied by physicochemical changes such as inorganic ion exchanges and serum proteins adsorption.

Combining OCP with natural polymers, such as collagen and gelatin, improves not only their moldabilities but also increases the osteoconductivity and the biodegradability in vivo. The hydrolysis of OCP may be involved in displaying bone regenerative capacity of OCP.

Keywords Octacalcium phosphate $(\mathrm{OCP}) \cdot$ Hydroxyapatite Hydrolysis · Osteoblasts · Osteoclasts · Bone substitute materials

\subsection{Introduction}

It has been advocated that octacalcium phosphate (OCP, $\left.\mathrm{Ca}_{8} \mathrm{H}_{2}\left(\mathrm{PO}_{4}\right)_{6} \cdot 5 \mathrm{H}_{2} \mathrm{O}\right)$ could be a precursor phase to biological apatite crystals in the bone (Brown 1966) although there is still a controversy about whether OCP is actually present

O. Suzuki $(\bowtie) \cdot$ T. Anada

Division of Craniofacial Function Engineering, Graduate School of Dentistry,

Tohoku University, Sendai, Japan

e-mail: suzuki-o@m.tohoku.ac.jp; anada@m.tohoku.ac.jp 
in bone mineralization (Rey et al. 2014). It has been reported that in a highly supersaturated calcium and phosphate solution with respect to hydroxyapatite ( $\left.\mathrm{HA}, \mathrm{Ca}_{10}\left(\mathrm{PO}_{4}\right)_{6}(\mathrm{OH})_{2}\right)$, amorphous calcium phosphate $\left(\mathrm{ACP}, \mathrm{Ca}_{3}\left(\mathrm{PO}_{4}\right)_{2} \cdot \mathrm{nH}_{2} \mathrm{O}\right)$ is formed first, and then it transforms to an OCP-like phase before crystallizing to the thermodynamically most stable HA phase (Meyer et al. 1978) at physiological $\mathrm{pH}$. ACP has been confirmed in growing chicken embryonic long bone (Kerschnitzki et al. 2016). The structure of the OCP-like phase has been suggested to resemble HA having a similar characteristics in X-ray diffraction (XRD) (Meyer et al. 1978). In fact, it has been shown that an apatite structure, having a solubility similar to OCP, can be synthesized in the presence of fluoride ions (Shiwaku et al. 2012). It has also been shown that ACP is aggregated as a cluster forms and then transforms to HA via a Ca-deficient OCP-like structure (Habraken et al. 2013) and that growing calcium phosphates involve the common cluster structure throughout the HA formation (Onuma et al. 2017). We hypothesized that the introduction of synthetic OCP into bone defects should lead to the enhancement of the initial deposition of bone matrix followed by additional bone formation (Suzuki et al. 1991). An experiment of onlay graft of OCP in the granule form onto mouse calvaria, in comparison with HA materials, was conducted to test the hypothesis and showed that OCP enhances the appearance of bone tissue around these granules more than those by HA materials (Suzuki et al. 1991). This review article summarizes how the OCP materials work in bone repair if placed in bone defects and in the vicinity of bone-tissue-related cells in vitro.

\subsection{Bone-Bonding Property of OCP Implanted in Bone Defects}

It has been proposed that direct bone bonding of ceramic materials is brought about through an apatite layer formation on the materials, thereby allowing chemical bonding between newly formed bone apatite crystals and the apatite formed on the materials (Kokubo 1991). Such direct bonding has been reported to occur in ceramic materials, such as glass ceramics (Hench et al. 1973; Kokubo 1991), HA (Aoki 1973 ), and $\beta$-tricalcium phosphate ( $\beta$-TCP, $\left.\mathrm{Ca}_{3}\left(\mathrm{PO}_{4}\right)_{2}\right)$ (Kotani et al. 1991). The property is ascribed to the osteoconduction, which is defined as the bone formation taking place in orthotopic site (LeGeros 2002). We have found that when the tissue response was observed using undecalcified sections of the granules of OCP implantation onto mouse calvaria by transmission electron microscopy, newly formed bone crystals directly bonded to the surface of crystals in the OCP granules (Suzuki et al. 2008). From this observation, it was apparent that the OCP has an osteoconductive property (Oyane et al. 2012). 


\subsection{Hydrolysis from OCP to Ca-Deficient HA in Physiological Conditions}

It is known that OCP is more soluble than $\beta$-TCP and HA and less soluble than dicalcium phosphate dihydrate (DCPD, $\left.\mathrm{CaHPO}_{4} \cdot 2 \mathrm{H}_{2} \mathrm{O}\right)$ under neutral $\mathrm{pH}$ (Brown et al. 1981; Chow 2009). It is of interest to learn about whether OCP is actually converted to HA in vivo conditions. It has been expected that OCP is converted (hydrolyzed) to HA if placed in the in vivo environment (Suzuki et al. 1991). There is a general consensus that OCP is stacked by apatite layer alternatively with hydrated layers and that the hydrolysis is once initiated; it advances spontaneously and irreversibly (LeGeros et al. 1989; Suzuki et al. 1995a, b; Tomazic et al. 1989). The structural changes of OCP have been investigated because the laboratory synthesized OCP is a well-grown crystal (Kobayashi et al. 2014; Sakai et al. 2016; Suzuki et al. 1991, 2006a, b). Other investigations showed that OCP remained untransformed in simulated body fluid (SBF) even prolonged incubation (Ito et al. 2014) under supersaturated conditions with respect to HA theoretically estimated (Lu et al. 2005). The implantation of OCP in the bone and subcutaneous tissues promoted the structural changes in XRD (Suzuki et al. 1991, 1993, 2006b) and in Fourier transform infrared (FTIR) absorption from that of OCP to apatite structure with increasing $\mathrm{Ca} / \mathrm{P}$ molar ratio (Sakai et al. 2016) and carbonate ion containment (Suzuki et al. 2009) although characteristics of OCP in XRD still remained (Sakai et al. 2016; Suzuki et al. 1991, 1993, 2006b). One of features in the structural changes of OCP by its implantation and incubation in physiological conditions is that the chemical composition of $\mathrm{Ca} / \mathrm{P}$ molar ratio has never reached to that of stoichiometric value (1.67) but tends to go to producing a Ca-deficiency, resulting in the formation of a Ca-deficient HA (Sakai et al. 2016; Suzuki et al. 1995b). OCP hydrolysis can be enhanced with experimentally given higher supersaturated conditions in vitro by promoting calcium ion consumption into the crystals and phosphate ion release from the crystals (Kobayashi et al. 2014; Sakai et al. 2016; Suzuki et al. 2006a), suggesting that in vivo environment could be providing such an ionic conditions. It has been reported that human serum is saturated with respect to OCP (Eidelman et al. 1987), which is not contradict to the proposition that HA can be grown on OCP template (Miake et al. 1993).

\subsection{Osteoblastic Cell Response}

It was observed that, when the granules of OCP were implanted onto mouse calvaria, the crystals of OCP were accumulated by circulating non-collagenous serum proteins, including $\alpha_{2}$ HS-glycoproteins and apolipoprotein E (Kaneko et al. 2011; Suzuki et al. 1993), the ultrastructure of which bears a close resemblance to the tissue structure so-called bone nodules, which is considered as the initial bone deposition locus in intramembranous bone development (Barradas et al. 2011; Suzuki 
et al. 1991). Osteoblasts then started to form a collagenous bone matrix around the bone nodule-like OCP-protein complex (Suzuki et al. 1991, 2008), indicating that OCP acts on a nucleus of bone formation. From these observations, it was hypothesized that OCP may activate osteoblastic cell activity on its surfaces. In order to test the hypothesis, mouse bone marrow stromal ST-2 cells were inoculated on OCP particles coated on plastic cell culture plate in comparison with HA materials (Anada et al. 2008; Suzuki et al. 2006b). mRNAs of osteoblast differentiation markers, such as alkaline phosphatase (ALP), type I collagen, and osterix, increased with increasing the dose of OCP (Anada et al. 2008).

\subsection{Osteoclastic Cell Response}

When OCP is placed in bone defects, OCP shows biodegradable characteristics that tend to be replaced with new bone (Honda et al. 2009; Imaizumi et al. 2006; Kikawa et al. 2009; Miyatake et al. 2009; Murakami et al. 2010; Suzuki 2013). It was ascertained that OCP hydrolysis is accompanied by a subtle reduction from a neutral $\mathrm{pH}$ to some extent acidic $\mathrm{pH}$ with the hydrolysis (Masuda et al. 2017), which may affect the cellular responses of immune cells (Hirayama et al. 2016). A histological examination showed that OCP enhanced macrophage migration (Hirayama et al. 2016) and multinucleated giant cells appearance around the surfaces (Honda et al. 2009; Imaizumi et al. 2006; Kikawa et al. 2009; Miyatake et al. 2009; Murakami et al. 2010; Suzuki 2013), where osteoblasts are forming new bone, more than HA (Suzuki 2013). The multinucleated giant cells were shown to be tartrate-resistant acid phosphatase (TRAP) positive osteoclast-like cells (Imaizumi et al. 2006), suggesting that OCP biodegrades through phagocytic resorption not by simple chemical dissolution. It is known that osteoclasts can be formed by the fusion of macrophages (Asagiri et al. 2007; Kong et al. 1999; Yasuda et al. 1999) so that the macrophage migration to OCP surfaces may be involved in the formation of osteoclast-like cells (Hirayama et al. 2016). An in vitro study in fact revealed that the multinucleated giant cells, expressing osteoclast marker genes such as TRAP and cathepsin K, can be formed on the surface of OCP but not on the surface of HA in the co-culture with osteoblasts (Takami et al. 2009). The osteoblasts cultured on OCP expressed receptor activator of NF-kappaB ligand (RANKL), an osteoclast differentiation factor (Takami et al. 2009). Calcium ion concentration in culture medium decreased in the presence of OCP, which corresponds to the change induced by OCP during its hydrolysis (Suzuki et al. 2006a), while the experimentally reduced calcium ion concentration induced RANKL mRNA expression of osteoblasts (Takami et al. 2009). These results suggest that the physicochemical environment induced by OCP brings about the biodegradable characteristics of this material through osteoclast differentiation from macrophages increasing through RANKL expressions by osteoblasts in the implanted OCP. The mechanism of physicochemical changes, including the protein adsorption, induced during the hydrolysis from 


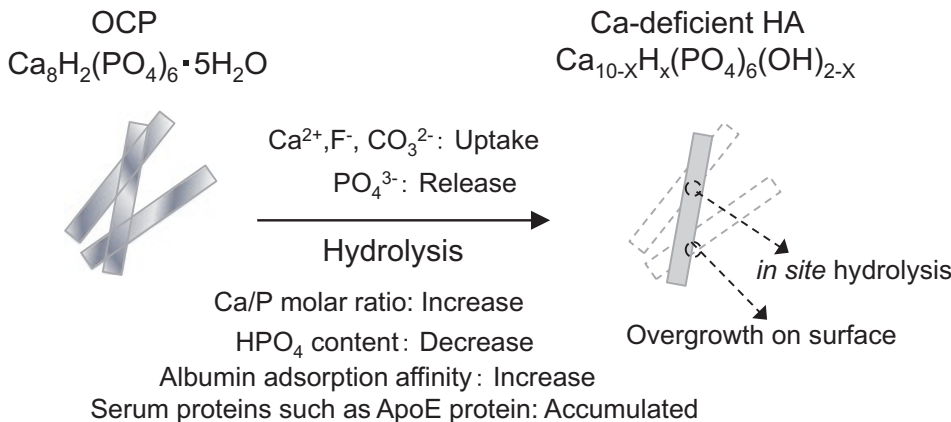

Fig. 20.1 Observed various physicochemical reactions caused during the hydrolysis of synthetic OCP placed in vivo(Suzuki et al. 1991, 2006b, 2008) and in vitro (Suzuki et al. 2006a, b) environments. OCP can be progressively hydrolyzed to Ca-deficient HA under a physiological environment via topotaxial conversion (in situ hydrolysis) and dissolution-reprecipitation (overgrowth of apatite on an OCP crystal surface) (Brown et al. 1981; Miyake et al. 1993; Suzuki et al. 1995b). Ca-deficient HA can be formed, and chemical composition change and surface reaction are promoted during the hydrolysis (Suzuki et al. 1995b, 2006b; Kaneko et al. 2011)

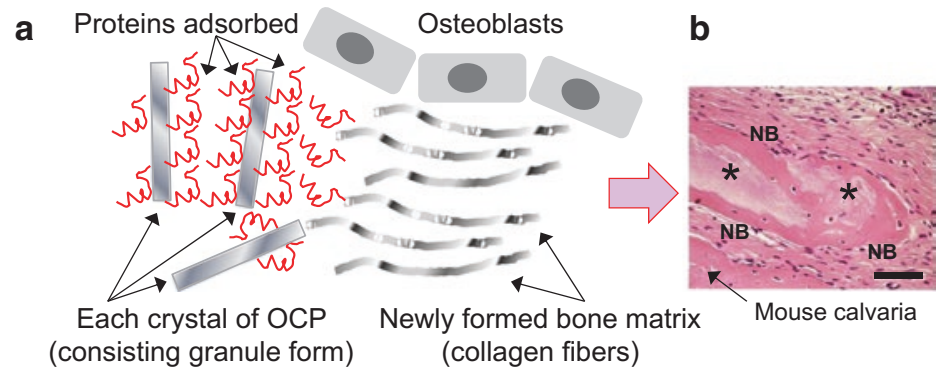

Fig. 20.2 Conceptual scheme of the beginning of bone formation from the synthetic OCP crystals implanted in bone tissue that is based on the ultrastructural observation (a) (Suzuki et al. 1991, 1993, 2008) and newly boned bone around OCP granules implanted onto mouse calvaria for 21 days (b). Right figure (b), decalcified histological section stained by hematoxylin and eosin. Asterisks (*): OCP granules, $N B$ new bone; bar $=50 \mu \mathrm{m}$. (Reproduced from Fig. $2 \mathrm{c}$ in Acta Biomater 5:1756-1766, 2009 (Kikawa et al. 2009) with permissions from Elsevier Ltd)

OCP to Ca-deficient HA, is summarized in Fig. 20.1. The osteoblast differentiation stimulated by OCP, as a nucleus of the initial bone deposition, is summarized in Fig. 20.2.

\subsection{Bone Substitute Materials}

OCP cannot be sintered with keeping its crystalline phase, unlike HA and $\beta$-TCP, due to the inclusion of large amount of water molecules (Brown et al. 1962). The granules or the precipitates of OCP were combined with various natural polymers, 
such as collagen, gelatin, alginate, and hyaluronic acid, in order to acquire the moldability and improve the handling property (Fuji et al. 2009; Handa et al. 2012; Kamakura et al. 2006; Shiraishi et al. 2010; Suzuki et al. 2014). OCP/collagen was made of OCP granules mixed with re-constituted collagen matrix (Kamakura et al. 2006). OCP/gelatins were made of OCP granules mixed with re-constituted gelatin matrix (Saito et al. 2016) and OCP directly precipitated with gelatin molecules (Handa et al. 2012). OCP/collagen composite enhanced the bone formation more than OCP alone when compared in a rat critical-sized calvaria defect (Kamakura et al. 2006). Bone regeneration was augmented in a dose-dependent manner of OCP in the collagen matrix (Kawai et al. 2009), which corresponded to the tendency of increasing osteoblastic cell differentiation in vitro (Anada et al. 2008). After preclinical trial (Kawai et al. 2014), the OCP/collagen composite is under a companyinitiative clinical trial in the field of oral surgery in Japan. The OCP-co-precipitated gelatin composite showed a highly biodegradable property in critical-sized rat calvaria defect (Handa et al. 2012) and also in rabbit tibia defect which is an orthopedic bone defect model (Chiba et al. 2016). The inclusion of OCP crystals in gelatin was shown to produce an oriented bone regarding newly formed collagen matrix (IshikoUzuka et al. 2017). OCP/alginates were made of OCP granules mixed with a calcium-cross-linked re-constituted alginate matrix (Shiraishi et al. 2010) and OCP directly precipitated with alginate molecules (Fuji et al. 2009). The combining OCP with alginate allowed the composite to proliferate osteoblastic cells although the alginate is a material that does not have a cellular binding motif (Fuji et al. 2009). OCP/hyaluronic acids (HyAs) were made of OCP granules mixed with HyA medical products, having different molecular weights (Suzuki et al. 2014). OCP/HyAs acquired injectability and OCP combined with a lower molecular weight HyA and with a higher molecular weight HyA enhanced bone augmentation more than OCP alone when placed on the subperiosteal region of mouse calvaria through an osteoclastic resorption of OCP (Suzuki et al. 2014). Thus, the osteoconductive property and the handling property of OCP could be controlled by those natural polymers that are combined with OCP.

\subsection{Conclusion}

It seems likely that the enhancement of bone formation by OCP implantation is derived from its stimulatory capacity on osteoblastic cell activity during its crystallizing into Ca-deficient HA. OCP-based materials could be used as bone substitute materials in various bone defects.

Acknowledgments This study was supported in part by grants-in-aid (17076001, 23106010 17K19740 and 18H02981) from the Ministry of Education, Science, Sports, and Culture of Japan, the Uehara Memorial Foundation, the Suzuken Memorial Foundation, and the Iketani Science and Technology Foundation. The author thanks Emeritus Professor and former President of Japan Fine Ceramics Co. Ltd., S. Ito; Professors R. Kamijo, M. Nakamura, and M. Takami, Showa University; 
Professor T. Katagiri, Saitama Medical School; Professors E. Itoi, S. Kamakura, T. Takahashi, and Emeritus; Professors M. Sakurai, M. Kagayama, and S. Echigo, Tohoku University; Drs. T. Kawai, and Y. Shiwaku, Tohoku University; and Dr. Y. Honda, Osaka Dental University.

\section{References}

Anada T et al (2008) Dose-dependent osteogenic effect of octacalcium phosphate on mouse bone marrow stromal cells. Tissue Eng Part A 14:965-978

Aoki H (1973) Synthetic apatite as an effective implant material Kokubyo Gakkai zasshi. J Stomatol Soc Jpn 40:277

Asagiri M et al (2007) The molecular understanding of osteoclast differentiation. Bone 40:251-264

Barradas AM et al (2011) Osteoinductive biomaterials: current knowledge of properties, experimental models and biological mechanisms. Eur Cell Mater 21:407-429 discussion 429

Brown W (1966) Crystal growth of bone mineral. Clin Orthop Relat Res 44:205-220

Brown WE et al (1962) Crystallographic and chemical relations between octacalcium phosphate and hydroxyapatite. Nature 196:1050-1055

Brown WE et al (1981) Crystal chemistry of octacalcium phosphate. Prog Cryst Growth Charact 4:59-87

Chiba S et al (2016) Effect of resorption rate and osteoconductivity of biodegradable calcium phosphate materials on the acquisition of natural bone strength in the repaired bone. J Biomed Mater Res A 104:2833-2842

Chow LC (2009) Next generation calcium phosphate-based biomaterials. Dent Mater J 28:1-10

Eidelman $\mathrm{N}$ et al (1987) Calcium phosphate saturation levels in ultrafiltered serum. Calcif Tissue Int 40:71-78

Fuji T et al (2009) Octacalcium phosphate-precipitated alginate scaffold for bone regeneration. Tissue Eng Part A 15:3525-3535

Habraken WJ et al (2013) Ion-association complexes unite classical and non-classical theories for the biomimetic nucleation of calcium phosphate. Nat Commun 4:1507

Handa $T$ et al (2012) The effect of an octacalcium phosphate co-precipitated gelatin composite on the repair of critical-sized rat calvarial defects. Acta Biomater 8:1190-1200

Hench LL et al (1973) Direct chemical bond of bioactive glass-ceramic materials to bone and muscle. J Biomed Mater Res 7:25-42

Hirayama B et al (2016) Immune cell response and subsequent bone formation induced by implantation of octacalcium phosphate in a rat tibia defect. RSC Adv 6:57475-57484

Honda Y et al (2009) The effect of microstructure of octacalcium phosphate on the bone regenerative property. Tissue Eng Part A 15:1965-1973

Imaizumi H et al (2006) Comparative study on osteoconductivity by synthetic octacalcium phosphate and sintered hydroxyapatite in rabbit bone marrow. Calcif Tissue Int 78:45-54

Ishiko-Uzuka R et al (2017) Oriented bone regenerative capacity of octacalcium phosphate/gelatin composites obtained through two-step crystal preparation method. J Biomed Mater Res B Appl Biomater 105:1029-1039

Ito $\mathrm{N}$ et al (2014) Importance of nucleation in transformation of octacalcium phosphate to hydroxyapatite. Mater Sci Eng C Mater Biol Appl 40:121-126

Kamakura $S$ et al (2006) Octacalcium phosphate combined with collagen orthotopically enhances bone regeneration. J Biomed Mater Res B Appl Biomater 79:210-217

Kaneko $\mathrm{H}$ et al (2011) Proteome analysis of rat serum proteins adsorbed onto synthetic octacalcium phosphate crystals. Anal Biochem 418:276-285

Kawai T et al (2009) Synthetic octacalcium phosphate augments bone regeneration correlated with its content in collagen scaffold. Tissue Eng Part A 15:23-32

Kawai T et al (2014) First clinical application of octacalcium phosphate collagen composite in human bone defect. Tissue Eng Part A 20:1336-1341 
Kerschnitzki M et al (2016) Bone mineralization pathways during the rapid growth of embryonic chicken long bones. J Struct Biol 195:82-92

Kikawa $\mathrm{T}$ et al (2009) Intramembranous bone tissue response to biodegradable octacalcium phosphate implant. Acta Biomater 5:1756-1766

Kobayashi K et al (2014) Osteoconductive property of a mechanical mixture of octacalcium phosphate and amorphous calcium phosphate. ACS Appl Mater Interfaces 6:22602-22611

Kokubo T (1991) Bioactive glass ceramics: properties and applications. Biomaterials 12:155-163

Kong Y et al (1999) OPGL is a key regulator of osteoclastogenesis, lymphocyte development and lymph-node organogenesis. Nature 397:315-323

Kotani $S$ et al (1991) Bone bonding mechanism of beta-tricalcium phosphate. J Biomed Mater Res 25:1303-1315

LeGeros RZ (2002) Properties of osteoconductive biomaterials: calcium phosphates. Clin Orthop Relat Res 395:81-98

LeGeros RZ et al (1989) Solution-mediated transformation of octacalcium phosphate (OCP) to apatite. Scan Electron Microsc 3:129-137 discussion 137-138

Lu X et al (2005) Theoretical analysis of calcium phosphate precipitation in simulated body fluid. Biomaterials 26:1097-1108

Masuda $\mathrm{T}$ et al (2017) Application of an indicator-immobilized-gel-sheet for measuring the $\mathrm{pH}$ surrounding a calcium phosphate-based biomaterial. J Biomater Appl 31:1296-1304

Meyer JL et al (1978) A thermodynamic analysis of the secondary transition in the spontaneous precipitation of calcium phosphate. Calcif Tissue Res 25:209-216

Miake Y et al (1993) Epitaxial overgrowth of apatite crystals on the thin-ribbon precursor at early stages of porcine enamel mineralization. Calcif Tissue Int 53:249-256

Miyatake $\mathrm{N}$ et al (2009) Effect of partial hydrolysis of octacalcium phosphate on its osteoconductive characteristics. Biomaterials 30:1005-1014

Murakami Y et al (2010) Comparative study on bone regeneration by synthetic octacalcium phosphate with various granule sizes. Acta Biomater 6:1542-1548

Onuma K et al (2017) Nanoparticles in $\beta$-tricalcium phosphate substrate enhance modulation of structure and composition of an octacalcium phosphate grown layer. CrystEngComm 19:6660-6672

Oyane A et al (2012) Calcium phosphate composite layers for surface-mediated gene transfer. Acta Biomater 8:2034-2046

Rey C et al (2014) What bridges mineral platelets of bone? Bonekey Rep 3:586

Saito K et al (2016) Dose-dependent enhancement of octacalcium phosphate biodegradation with a gelatin matrix during bone regeneration in a rabbit tibial defect model. RSC Adv 6:64165-64174

Sakai S et al (2016) Comparative study on the resorbability and dissolution behavior of octacalcium phosphate, beta-tricalcium phosphate, and hydroxyapatite under physiological conditions. Dent Mater J 35:216-224

Shiraishi N et al (2010) Preparation and characterization of porous alginate scaffolds containing various amounts of octacalcium phosphate (OCP) crystals. J Mater Sci Mater Med 21:907-914

Shiwaku Y et al (2012) Structural, morphological and surface characteristics of two types of octacalcium phosphate-derived fluoride-containing apatitic calcium phosphates. Acta Biomater 8:4417-4425

Suzuki O (2013) Octacalcium phosphate (OCP)-based bone substitute materials. Jpn Dent Sci Rev 49:58-71

Suzuki O et al (1991) Bone formation on synthetic precursors of hydroxyapatite. Tohoku J Exp Med 164:37-50

Suzuki O et al (1993) Maclura pomifera agglutinin-binding glycoconjugates on converted apatite from synthetic octacalcium phosphate implanted into subperiosteal region of mouse calvaria. Bone Miner 20:151-166

Suzuki O et al (1995a) Reversible structural changes of octacalcium phosphate and labile acid phosphate. J Dent Res 74:1764-1769 
Suzuki O et al (1995b) Adsorption of bovine serum albumin onto octacalcium phosphate and its hydrolyzates. Cell Mater 5:45-54

Suzuki O et al (2006a) Surface chemistry and biological responses to synthetic octacalcium phosphate. J Biomed Mater Res B Appl Biomater 77:201-212

Suzuki O et al (2006b) Bone formation enhanced by implanted octacalcium phosphate involving conversion into Ca-deficient hydroxyapatite. Biomaterials 27:2671-2681

Suzuki O et al (2008) Bone regeneration by synthetic octacalcium phosphate and its role in biological mineralization. Curr Med Chem 15:305-313

Suzuki Y et al (2009) Appositional bone formation by OCP-collagen composite. J Dent Res $88: 1107-1112$

Suzuki K et al (2014) Effect of addition of hyaluronic acids on the osteoconductivity and biodegradability of synthetic octacalcium phosphate. Acta Biomater 10:531-543

Takami M et al (2009) Osteoclast differentiation induced by synthetic octacalcium phosphate through RANKL expression in osteoblasts. Tissue Eng Part A 15:3991-4000

Tomazic BB et al (1989) Mechanism of hydrolysis of octacalcium phosphate. Scanning Microsc 3:119-127

Yasuda $\mathrm{H}$ et al (1999) A novel molecular mechanism modulating osteoclast differentiation and function. Bone 25:109-113

Open Access This chapter is licensed under the terms of the Creative Commons Attribution 4.0 International License (http://creativecommons.org/licenses/by/4.0/), which permits use, sharing, adaptation, distribution and reproduction in any medium or format, as long as you give appropriate credit to the original author(s) and the source, provide a link to the Creative Commons license and indicate if changes were made.

The images or other third party material in this chapter are included in the chapter's Creative Commons license, unless indicated otherwise in a credit line to the material. If material is not included in the chapter's Creative Commons license and your intended use is not permitted by statutory regulation or exceeds the permitted use, you will need to obtain permission directly from the copyright holder.

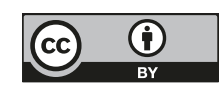

